\title{
Persone come noi. La testimonianza di Alex: quando la terapia a domicilio fa la differenza
}

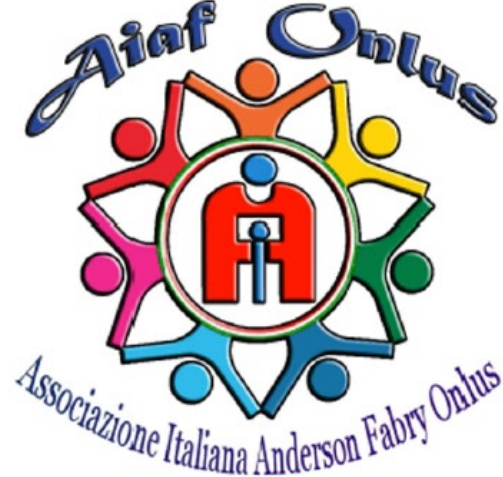

Nel corso del convegno "Home Therapy, per una migliore qualità di vita”, tenutosi a Roma lo scorso 24 ottobre, è stata proiettata una video-intervista ad Alex, un giovane con Malattia di Anderson-Fabry, che attraverso il proprio racconto ha permesso di far comprendere meglio l'impatto della terapia sulla quotidianità. Nelle sue parole è riassunta la difficile situazione che sono costretti a subire molti pazienti affetti da malattie rare lisosomiali.

"Mi chiamo Alex, ho 27 anni e gestisco un Bed\&Breakfast nella mia città. E' un lavoro che mi permette di convivere con i miei problemi di salute e con la mia malattia di Anderson-Fabry, una malattia da accumulo lisosomiale che provoca parestesie, dolori molto acuti a mani e piedi, problemi neurologici e in alcuni casi compromissioni a cuore, reni e organi interni.

Da bambino ho cominciato ad avvertire i primi sintomi. Poi il doloroso percorso a ping-pong ospedaliero, che mi ha portato per anni da un ospedale ad un altro alla ricerca di una diagnosi. Sono riuscito a sapere il nome della mia malattia solo quando avevo 11 anni. Da allora è iniziata la ricerca della terapia più adeguata, che è risultata la terapia enzimatica sostitutiva. Una terapia che avrei dovuto effettuare per tutta la vita.

La terapia mi viene somministrata per infusione ogni due settimane in un ospedale a 50 chilometri da casa. Questo ha significato per me e per la mia famiglia dover affrontare ogni volta un viaggio di 100 chilometri tra andata e ritorno. Un problema che ha continuato a presentarsi anche quando ho preso la patente di guida: guidare, soprattutto per tragitti così lunghi, è per me molto doloroso. Per non parlare delle ore, delle giornate trascorse solo per ricevere la terapia, e, talvolta, dell'impossibilità di mantenere la continuità nella somministrazione. Quando
Giornale di Tecniche Nefrologiche e Dialitiche 2018, Vol. 30(3-4) 234

(C) The Author(s) 2019

Article reuse guidelines:

sagepub.com/journals-permissions DOI: I0.1 I77/0394936219832320

journals.sagepub.com/home/gtn

@SAGE

mi sono iscritto all'università, fuori dalla mia regione, ho dovuto fare il pendolare per continuare a ricevere la terapia.

Nel corso del secondo anno di università sono finalmente riuscito ad ottenere la possibilità di effettuare la terapia a domicilio. Un sogno! Si concretizzava finalmente la possibilità di avere una vita normale. Fare le infusioni a casa è come entrare in un altro mondo! Ti si cuciono addosso gli orari, si può fare una vacanza, si può viaggiare. Evitare di doverti recare in ospedale e avere i tuoi cari intorno mentre fai l'infusione, oppure aiutare tua moglie, che può andare a lavorare mentre tu puoi tenere tuo figlio anche se stai facendo la terapia: sono queste le piccole cose che trasformano e migliorano la vita di una persona già duramente provata da una malattia. Non solo. C'è un'intera equipe che ti segue: un'infermiera che ha una comunicazione diretta e continua con lo specialista, e tutti lavorano esclusivamente per te. In ospedale, invece, seguono decine e decine di persone che stanno come te, se non peggio. In più, non è vero che la terapia effettuata in casa offre meno controllo, anzi! Il controllo aumenta perché tu non sei solo uno dei tanti, sei Alex, sei la persona malata che in quel momento viene seguita e trattata, in ogni piccolo dettaglio.

Ma poi una telefonata ha cambiato tutto. Mi hanno cacciato dal paradiso. Per un problema burocratico, mi è stato tolto il servizio a domicilio e sono stato reinviato alla terapia in ospedale. Ho dovuto riassestare di nuovo la mia vita intorno alla cura. Ho dovuto di nuovo chiedere aiuto alla mia famiglia, con molte difficoltà perché anche loro hanno impegni e non sempre possono essere a mia disposizione, nonostante io non riesca a guidare.

Per qualche tempo, grazie alla disponibilità dei medici e delle strutture ospedaliere, ero riuscito a non sentirmi più ammalato, ma la malattia è poi ritornata con prepotenza nella mia vita, per colpa della burocrazia.

Sono una persona che reagisce, che lotta, ma in quel periodo ho avuto un crollo e mi sono lasciato andare. Sono anche arrivato a pensare: "basta, lascio stare!".

Ora mi auguro che le autorità sanitarie rivedano la loro decisione: fare la terapia a casa cambierebbe la vita a me e a tutti i pazienti in maniera....EPICA! “

(Video intervista realizzata a cura di Adn Kronos per Aiaf Onlus) 